\title{
Angela Gotthardt-Lorenz: Organisationssupervision - ein Konzept. Göttingen: Vandenhoek \& Ruprecht 2020, 105 S.
}

\section{Eva Senghaas-Knobloch ${ }^{1}$}

Angenommen: 15. April 2021 / Online publiziert: 4. Oktober 2021 (c) Der/die Autor(en) 2021

Die Wiener Supervisorin und Lehrsupervisorin Angela Gotthardt-Lorenz, hat in dem Band ihr Konzept zur Organisationssupervision theoretisch und konzeptionell erweitert und fundiert. Sie nutzt Erkenntnisse der subjektorientierten Arbeitssoziologie, um das komplexe Geschehen in Organisationen in einem Drei-Ebenen-Modell zu systematisieren und Ansatzpunkte für Supervision zu empfehlen.

Auf der ersten Ebene geht es um das, was Organisation allgemein ausmacht und um Organisationsstrukturen und -dynamiken, die auch von gesellschaftlichen und wirtschaftspolitischen Entwicklungen geprägt sind. Als konstituierend für Organisationssupervision werden die Anforderungen an Fachlichkeit und organisationale Angebote an soziale Zugehörigkeit genannt, herausgefordert durch begrenzte Ressourcen, bzw. atypische Beschäftigungsformen.

Auf der zweiten Ebene nutzt die Autorin Konzepte der Arbeits- und Organisationssoziologie, um die Ansatzpunkte für das Supervisionshandeln detailliert zu beschreiben. Dazu gehören vor allem das Konzept der sozialen Erwartungsstrukturen (G. Becke), das Konzept der Interaktionsarbeit, das im Forschungskontext um F. Böhle, W. Dunkel, M. Weihrich entstand, das Konzept professionellen Handelns auf Basis eines „Arbeitsbündnisses“ (Demszky/Voss) und das Gestaltungskonzept organisationaler Achtsamkeit, das G. Becke mit P. Bleses auf Basis von Sutcliffe und Weick weiterentwickelt hat.

An die supervisorische Praxis in Organisationen sind diese Konzepte besonders mit Blick auf notwendige Kooperationsbeziehungen anschlussfähig. Diese sind bei der personalen Dienstleistung zentral für die Auftragserfüllung durch Interaktionsarbeit, sei es mit Patienten, Kundinnen oder Klienten. Dabei geht es um Beachtung von notwendiger Gefühls- und Emotionsarbeit, von Ungewissheiten, Unwägbarkeiten sowie von neuen organisationalen An-

Eva Senghaas-Knobloch

esk@uni-bremen.de

1 Universität Bremen, Bremen, Deutschland forderungen an Formalisierung und Objektivierung, auch angesichts mangelnder Ressourcen. Ein hoher Stellenwert kommt ebenso den Interaktionsbeziehungen im innerorganisationalen Koordinations- und Kooperationsgeschehen zu, sowohl im kollegialen Verhältnis als auch im Verhältnis zwischen Fachkräften und Führungskräften. Hervorgehoben werden auch die arbeitssoziologisch beschriebenen Spannungen zwischen fachlichem Anspruch und deren Begrenzungen vor allem durch (Zeit-)Ressourcenmangel und zwischen dem emotional bedeutenden Angebot für organisationale/soziale Zugehörigkeit und deren Begrenzung, z.B. durch zeitliche Befristung.

Das soziologische Konzept sozialer Erwartungsstrukturen gilt der Autorin als anschlussfähig, weil so das in der Supervision geläufige dynamische Rollenkonzept mit dem statischen Begriff der Position innerhalb von Organisationen verbunden werden kann. An organisationale Positionen sind bestimmte formale und nicht formal gefasste Erwartungen geknüpft, z. B. über ein faires Geben und Nehmen zwischen Management und Mitarbeitenden. Dies ist ein Feld häufiger Enttäuschungen und Konflikte. Rollengestaltung auf organisationalen Positionen setzt Rollendistanz voraus, die es erlaubt, das eigene Handeln zu reflektieren.

Die arbeitssoziologischen Konzepte und Erkenntnisse in Organisationen nutzt die Autorin, um auf der dritten Ebene nun selbstreflexiv und methodisch-didaktisch die Aufgaben, Anforderungen und Kontexte der Supervisions- und Beratungssysteme in und für Organisationen zu beschreiben. Geht es z. B. darum, die Interaktionsräume, die von Supervision in Organisationen zur Verfügung gestellt wird, auf reflektierte Weise mitzugestalten, gehört dazu die Entwicklung adäquater Beratungssysteme angesichts wahrgenommener Erwartungsstrukturen. Der Interaktionsraum, den Organisationssupervision zur Verfügung stellt, ist besonders bedeutsam für sozio-emotionale Themen, so dass auch ,unverdauliche“ Affekte (Tietel) aufgenommen und transformiert werden können. Besonders wichtig ist Gotthardt-Lorenz die Unterstützung professionellen Handelns in Organisationen, wobei Supervision in der Spannung 
zwischen Professions- und Organisationslogik einen Platz finden muss. Dementsprechend erarbeitet die Autorin am Schluss eine fruchtbare Systematik zu Rollenerwartungen und Rollenangeboten.

Der Band dokumentiert die Fruchtbarkeit eines Austauschs zwischen arbeitssoziologischer Forschung und Supervision/Beratung. Angela Gotthardt-Lorenz ist es gelungen, tragfähige Brücken zwischen den zwei verschiedenen Professionen zu bauen. Dazu bedurfte es gemeinsamer Gelegenheitsstrukturen, die von beiden Seiten für die Kommunikation genutzt wurden, aber auch des Anstoßes durch den Bremer Psychologen und Supervisor Erhard Tietel, selbst ein Grenzgänger und Brückenbauer. Es ist zu wünschen, dass auch umgekehrt die subjektorientierte Arbeitsforschung eine Sensibilität für sozio-emotionale Verstrickungen in der Feldforschung ausbaut und - wo angesagt - Supervision und Intervision in die eigene Forschungsplanung einbezieht.

Funding Open Access funding enabled and organized by Projekt DEAL.
Open Access Dieser Artikel wird unter der Creative Commons Namensnennung 4.0 International Lizenz veröffentlicht, welche die Nutzung, Vervielfältigung, Bearbeitung, Verbreitung und Wiedergabe in jeglichem Medium und Format erlaubt, sofern Sie den/die ursprünglichen Autor(en) und die Quelle ordnungsgemäß nennen, einen Link zur Creative Commons Lizenz beifügen und angeben, ob Änderungen vorgenommen wurden.

Die in diesem Artikel enthaltenen Bilder und sonstiges Drittmaterial unterliegen ebenfalls der genannten Creative Commons Lizenz, sofern sich aus der Abbildungslegende nichts anderes ergibt. Sofern das betreffende Material nicht unter der genannten Creative Commons Lizenz steht und die betreffende Handlung nicht nach gesetzlichen Vorschriften erlaubt ist, ist für die oben aufgeführten Weiterverwendungen des Materials die Einwilligung des jeweiligen Rechteinhabers einzuholen.

Weitere Details zur Lizenz entnehmen Sie bitte der Lizenzinformation auf http://creativecommons.org/licenses/by/4.0/deed.de.

Eva Senghaas-Knobloch Prof. (em.) Dr. phil., Forschungszentrum Nachhaltigkeit; interdisziplinär orientierte Arbeits-, Friedens- und Konfliktforscherin mit dem Schwerpunkt Humanisierung des Arbeitslebens. 